\title{
Ultrahigh-efficiency apodized grating coupler using fully etched photonic crystals
}

\author{
Ding, Yunhong; Ou, Haiyan; Peucheret, Christophe
}

Published in:

Optics Letters

Link to article, DOI:

10.1364/OL.38.002732

Publication date:

2013

Document Version

Publisher's PDF, also known as Version of record

Link back to DTU Orbit

Citation (APA):

Ding, Y., Ou, H., \& Peucheret, C. (2013). Ultrahigh-efficiency apodized grating coupler using fully etched photonic crystals. Optics Letters, 38(15), 2732-2734. https://doi.org/10.1364/OL.38.002732

\section{General rights}

Copyright and moral rights for the publications made accessible in the public portal are retained by the authors and/or other copyright owners and it is a condition of accessing publications that users recognise and abide by the legal requirements associated with these rights.

- Users may download and print one copy of any publication from the public portal for the purpose of private study or research.

- You may not further distribute the material or use it for any profit-making activity or commercial gain

- You may freely distribute the URL identifying the publication in the public portal

If you believe that this document breaches copyright please contact us providing details, and we will remove access to the work immediately and investigate your claim 


\title{
Ultrahigh-efficiency apodized grating coupler using fully etched photonic crystals
}

\author{
Yunhong Ding, * Haiyan Ou, and Christophe Peucheret \\ Department of Photonics Engineering, Technical University of Denmark, 2800 Kgs. Lyngby, Denmark \\ ${ }^{*}$ Corresponding author: yudin@fotonik.dtu.dk \\ Received May 16, 2013; revised June 21, 2013; accepted July 1, 2013; \\ posted July 1, 2013 (Doc. ID 190742); published July 24, 2013
}

\begin{abstract}
We present an efficient method to design apodized grating couplers with Gaussian output profiles for efficient coupling between standard single mode fibers and silicon chips. An apodized grating coupler using fully etched photonic crystal holes on the silicon-on-insulator platform is designed, and fabricated in a single step of lithography and etching. An ultralow coupling loss of $-1.74 \mathrm{~dB}$ ( $67 \%$ coupling efficiency) with a $3 \mathrm{~dB}$ bandwidth of $60 \mathrm{~nm}$ is experimentally measured. (c) 2013 Optical Society of America

OCIS codes: (130.0130) Integrated optics; (230.3120) Integrated optics devices; (230.1950) Diffraction gratings; (050.2770) Gratings.

http://dx.doi.org/10.1364/OL.38.002732
\end{abstract}

Silicon-on-insulator (SOI) is considered as one of the most promising platforms for photonic integrated circuits thanks to its complementary metal-oxide semiconductor (CMOS) compatible fabrication technology [1] ]. In addition, the high refractive index contrast enables compact sizes for SOI devices. However, it also introduces a large mode mismatch between SOI nano waveguides and standard single mode fibers (SSMFs), making it very challenging to couple light between them efficiently. One method is to use a silicon inverse nano taper covered by a medium index contrast waveguide (such as polymer or silicon nitride) as a spot size converter [르료. However, this approach requires extra fabrication steps for the medium index contrast waveguide, and a lensed fiber is normally needed to reduce the mode mismatch. Another efficient solution is to use grating couplers. By properly designing the period and duty cycle of a diffraction grating structure implemented in a silicon waveguide, light guided in the waveguide can be coupled to an SSMF at a proper angle [4-10]. The grating coupler solution offers the great convenience of avoiding cleaving the sample, making wafer-scale testing possible. Normally the grating coupler is designed as uniform type with shallow etched slots to introduce a proper scattering strength [ [4-6] . However, the exponentially decaying field profiles of light diffracted from those gratings result in intrinsic mode mismatches to SSMFs with Gaussianlike field profiles, and the coupling efficiency is also sensitive to the etching depth [4]. In order to decrease such mode mismatches, shallowly etched apodized gratings have been proposed and demonstrated $[\underline{7}, \underline{8}]$, at the expense of extra processing steps. To simultaneously simplify the fabrication and avoid the etching depth sensitivity, fully etched grating couplers are preferred, so that silicon nanowires and the grating coupler can be fabricated in the same process. A uniform grating coupler based on fully etched photonic crystals (PhCs) has been demonstrated [9], exhibiting a coupling efficiency of $42 \%$. A fully etched apodized grating coupler using square holes have been realized [10] to reduce the mode mismatch, showing, however, only a slightly improved coupling loss of $-3.6 \mathrm{~dB}$ (44\% coupling efficiency).
In this paper, we present an efficient method to design an ultrahigh-efficiency apodized grating coupler. Using this method, a fully etched apodized grating coupler is designed and demonstrated on the SOI platform using $\mathrm{PhCs}$ to engineer the refractive index of each scattering slot. An ultralow coupling loss of $-1.74 \mathrm{~dB}$ ( $67 \%$ coupling efficiency) with a $3 \mathrm{~dB}$ bandwidth of $60 \mathrm{~nm}$ is demonstrated. The fabrication tolerance is also investigated, showing a $10 \mathrm{~nm}$ peak coupling wavelength shift for $4 \mathrm{~nm}$ hole size change.

The proposed method comprises four steps. The first step is to use a two-dimensional (2D) model to analyze the scattering cells of an apodized grating coupler (see Fig. 1) considering the buried silicon oxide (BOX) layer to be infinitely thick. As an example, a scattering angle $\theta=10^{\circ}$ is chosen and the grating coupler is designed to work on the transverse electric (TE) mode of the silicon waveguide. A $250 \mathrm{~nm}$ top silicon layer is considered, and air is used as upper cladding. For each scattering cell of length $l_{i}$, where the index $i$ denotes the number of the concerned cell, an artificial material slot with index $n_{i}$, and a slot width $d_{i}$ is introduced, resulting in a power leakage factor $2 \alpha_{i}$. In the design, $d_{i}$ is fixed to be $d_{0}=345 \mathrm{~nm}$ (inset of Fig. 2) so that $n_{i}$ can be tuned by only changing the PhC hole size, as described in step 4. The Bloch constant, which determines the effective refractive index $n_{\text {eff }}$ and power leakage factor $2 \alpha$, is calculated by the 2D eigenmode expansion method (EME) [11]. The scattering wavelength $\lambda$ is then given by

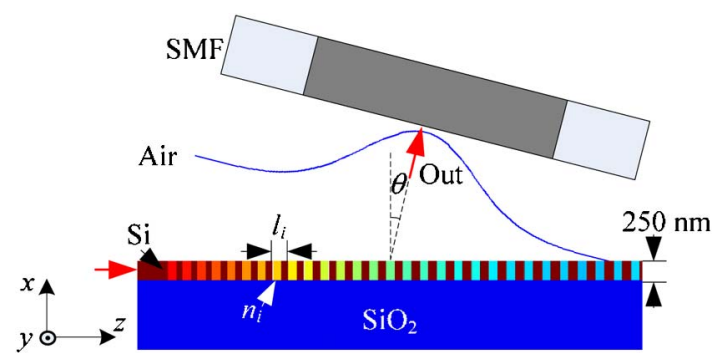

Fig. 1. 2D analytical model of the apodized grating coupler; SMF, single mode fiber. 


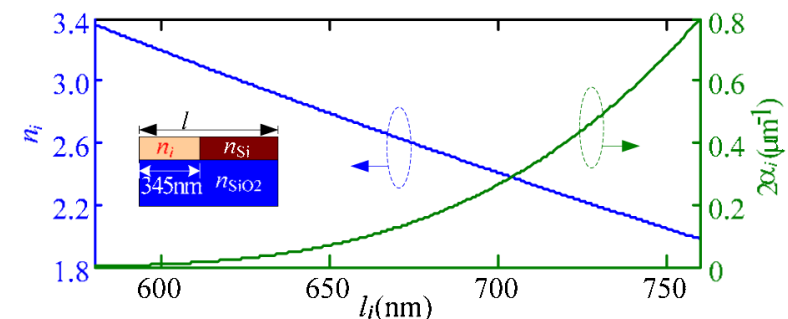

Fig. 2. Calculated required refractive index $n_{i}$ of the artificial material of a scattering cell and the corresponding power leakage factor as a function of the scattering cell length $l_{i}$.

$$
\lambda=l\left(n_{\mathrm{eff}}-n_{\mathrm{up}} \sin \theta\right),
$$

where $n_{\text {up }}$ is the refractive index of the upper cladding (air). For a given $l_{i}$, the required refractive index $n_{i}$ of the artificial material is found so that the scattering cell has a scattering angle $\theta=10^{\circ}$ at $1550 \mathrm{~nm}$. The corresponding $2 \alpha_{i}$ is also calculated, as shown in Fig. 2. This provides a guideline to synthesize any leakage factor distribution for a grating coupler.

The second step is to synthesize the required leakage factor distribution according to the results obtained in step 1. In order to obtain a Gaussian output profile with beam diameter of $10.4 \mu \mathrm{m}$, corresponding to that of an SSMF, the power leakage factor distribution $2 \alpha(z)$ of an apodized grating coupler should satisfy the following relation [8]:

$$
2 \alpha(z)=G^{2}(z) /\left[1-\int_{0}^{z} G^{2}(z) \mathrm{d} z\right] .
$$

Figure 3(a) shows the required power leakage factor, calculated according to Eq. (2), in order for a grating coupler to scatter a Gaussian beam centered at $z=10 \mu \mathrm{m}$. The grating parameters $l_{i}$ and $n_{i}$ are then designed according to the target power leakage profile [see Fig. 3(a)] and the calculated results of Fig. 2, as shown in Fig. 3(b), resulting in a good match with the target power leakage factor $2 \alpha(z)$ and the output Gaussian profile $G(z)$.

The third step is to optimize the BOX thickness with the designed grating parameters given by Fig. 3(b). The coupling efficiency is calculated by the $2 \mathrm{D} \overline{\mathrm{EME}}$ method as a function of the BOX thickness, as shown

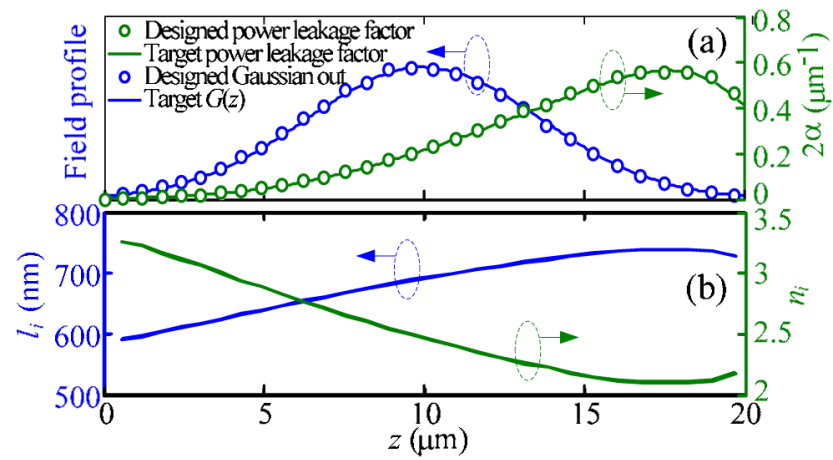

Fig. 3. (a) Designed and target power leakage factors and Gaussian output profiles. (b) Final dimension and refractive index distribution of the artificial material for the designed grating coupler.

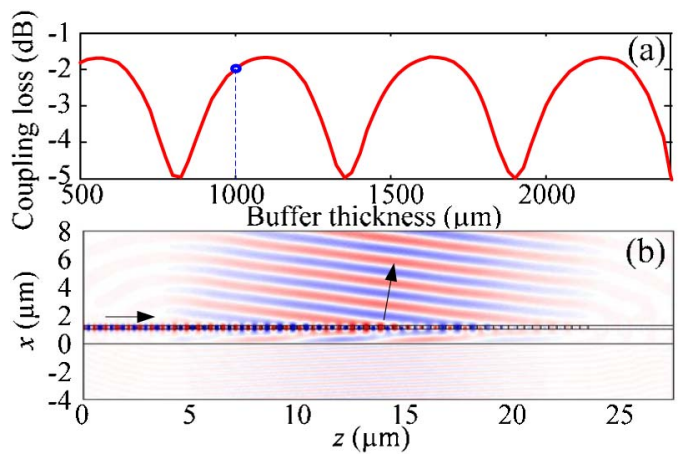

Fig. 4. (a) Calculated coupling efficiency as a function of the BOX thickness and (b) calculated field distribution $E_{y}$ at $1550 \mathrm{~nm}$, for the designed grating coupler with $1 \mu \mathrm{m}$ BOX layer.

in Fig. 4(a). One can find that the coupling efficiency depends periodically on the BOX thickness, and reaches its maximum with a BOX layer of around $1.1 \mu \mathrm{m}$. Figure 4(b) shows the calculated field distribution of the designed SOI grating coupler with a $1 \mu \mathrm{m}$ BOX layer when TE light is injected into the grating coupler, confirming that a Gaussian beam is vertically coupled out at $10^{\circ}$, as designed.

The final step is to engineer the refractive index of each artificial material slot using fully etched PhCs holes. In particular, a triangular lattice is selected in our design [9]. The effective index $n_{2 D}$ of a $2 \mathrm{D}$ triangular lattice $\mathrm{PhC}$ can be estimated as

$$
\frac{n_{2 D}^{2}}{n_{h}^{2}}=\frac{n_{c}^{2}(1+f)+n_{h}^{2}(1-f)}{n_{c}^{2}(1-f)+n_{h}^{2}(1+f)}
$$

where $n_{c}$ and $n_{h}$ are the refractive indices of the cylindrical inclusions (air) and host medium (silicon), respectively [12]. $f=3 \sqrt{3} \pi D^{2} / 16 d_{0}^{2}$ is the volume filling fraction of the cylindrical inclusions. The hole diameter $D$ of each artificial material slot is then determined by the required $n_{i}(z)$ distribution and Eq. (2). The final grating design is presented in Fig. 5(a). Figure 5(b) shows the calculated coupling efficiency of the designed grating coupler $(1 \mu \mathrm{m}$ BOX layer) as a function of wavelength.

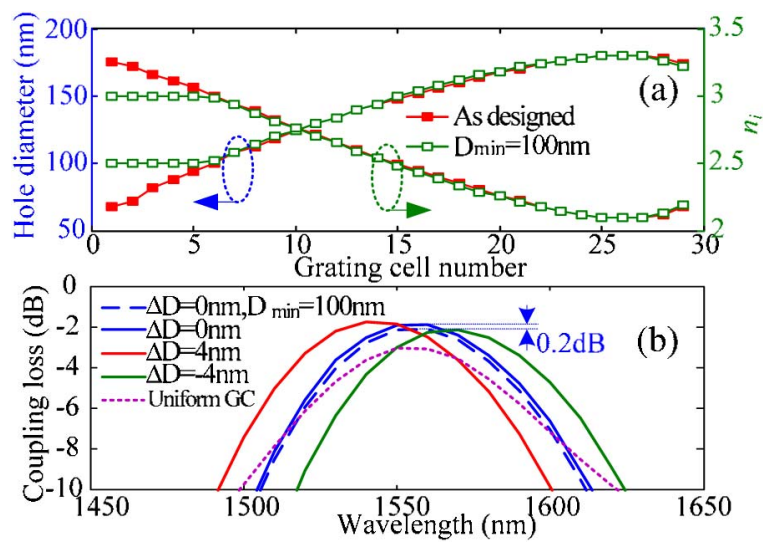

Fig. 5. (a) Grating coupler design with the original parameters and when the hole diameter is restricted to values above $100 \mathrm{~nm}$. (b) Corresponding calculated coupling efficiencies for uniform and optimized grating couplers with a $\pm 4 \mathrm{~nm}$ hole diameter deviation. 

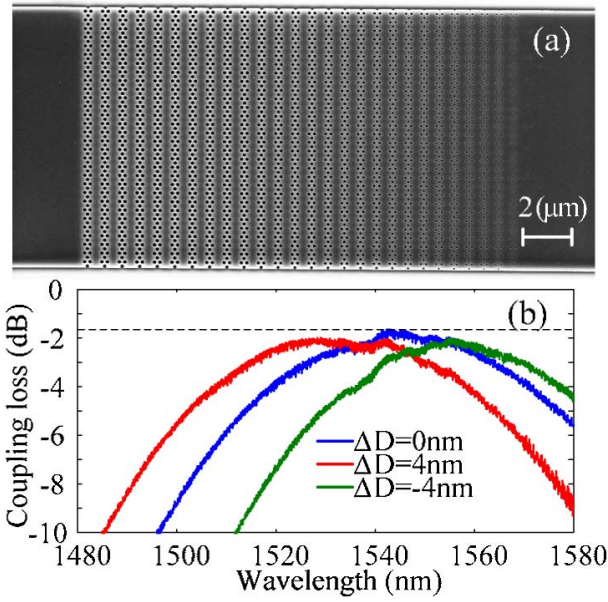

Fig. 6. (a) Fabricated apodized grating coupler. (b) Measured coupling efficiencies as a function of wavelength for the grating couplers with and without $\mathrm{a} \pm 4 \mathrm{~nm}$ hole diameter deviation.

A lowest coupling loss of $-1.8 \mathrm{~dB} \quad(66 \%$ coupling efficiency) is predicted, which is better than a calculated $-3.1 \mathrm{~dB}$ coupling loss for a uniform type grating with the same artificial slot width $d_{0}$ and an optimized $2 \alpha$ of $0.26 \mu \mathrm{m}^{-1}$ [13]. In addition, a hole size change of $\Delta D=$ $\pm 4 \mathrm{~nm}$ results in a $\pm 10 \mathrm{~nm}$ peak coupling wavelength shift. Considering that the minimum hole diameter $\left(D_{\min }\right)$ of our design is around $60 \mathrm{~nm}$, which could not be realized by fabrication methods such as deep ultraviolet (DUV) lithography, the impact of restricting the feasible hole diameter to above $100 \mathrm{~nm}$ is investigated, as shown in Fig. 5(a). In this case, the corresponding cell length should also be adjusted according to Fig. 2, since the effective refractive index $n_{i}$ of the slots with designed air hole diameters below $100 \mathrm{~nm}$ has been changed. One can find that only $0.2 \mathrm{~dB}$ degradation in maximum coupling loss is achieved, as shown in Fig. 5(b), indicating that the design is feasible with most fabrication methods.

In order to validate our design method, a grating coupler with the designed parameters was fabricated on a SOI wafer with top silicon thickness of $250 \mathrm{~nm}$ and BOX of $1 \mu \mathrm{m}$. A single step of standard $E$-beam lithography based nano fabrication processes [14] were used to fabricate the fully etched silicon wave-guides and grating couplers, simultaneously. The $450 \mathrm{~nm}$-wide and $700 \mu \mathrm{m}$ long straight waveguide is laterally tapered with $500 \mu \mathrm{m}$ tapering lengths at both ends to the grating couplers (20 $\mu \mathrm{m}$ long and $12 \mu \mathrm{m}$ wide). Several PhC columns with apodized hole sizes are introduced as the artificial material, as shown in Fig. 6(a). Figure 6(b) shows the measured coupling loss to an SSMF mounted at $10^{\circ}$ as a function of wavelength. The coupling loss is calculated as $\left(\eta_{0}-\eta_{s}\right) / 2$, where $\eta_{0}$ is the grating-to-grating transmission and $\eta_{s}$ is the loss of the straight wave-guide with a typical propagation loss of $4.3 \mathrm{~dB} / \mathrm{cm}$ [15]. A coupling loss of only $-1.74 \mathrm{~dB}$ ( $67 \%$ coupling efficiency) with a $3 \mathrm{~dB}$ bandwidth of $60 \mathrm{~nm}$ are measured. Grating couplers with a $\pm 4 \mathrm{~nm}$ hole diameter deviation are also characterized, showing $\pm 10 \mathrm{~nm}$ peak coupling wavelength shift, in good agreement with the calculations. Such a peak coupling wavelength shift can be compensated by adjusting the coupling angle [4]. To the best of our knowledge, this is the highest reported coupling efficiency for fully etched grating couplers. Further coupling loss improvement could be achieved with backside metal mirrors [16].

We have presented an efficient method to design an apodized grating coupler. An ultrahigh-efficiency fully etched apodized grating coupler based on PhCs has been designed and demonstrated, showing a low coupling loss of $-1.74 \mathrm{~dB}$ with a $3 \mathrm{~dB}$ bandwidth of $60 \mathrm{~nm}$. The fabrication tolerance of the designed grating coupler was also investigated both theoretically and experimentally.

\section{References}

1. T. Tsuchizawa, K. Yamada, H. Fukuda, T. Watanabe, J. Takahashi, M. Takahashi, T. Shoji, E. Tamechika, S. Itabashi, and H. Morita, IEEE J. Sel. Top. Quantum Electron. 11, 232 (2005).

2. V. R. Almeida, R. R. Panepucci, and M. Lipson, Opt. Lett., 28, 1302 (2003).

3. T. Tsuchizawa, K. Yamada, H. Fukuda, T. Watanabe, S. Uchiyama, and S. Itabashi, Jpn. J. Appl. Phys. 456658 (2006).

4. D. Taillaert, W. Bogaerts, P. Bienstman, T. F. Krauss, P. van Daele, I. Moerman, S. Verstuyft, K. Mesel, and R. Baets, IEEE J. Quantum Electron. 38, 949 (2002).

5. D. Taillaert, F. van Laere, M. Ayre, W. Bogaerts, D. van Thourhout, P. Bienstman, and R. Baets, Jpn. J. Appl. Phys. 45, 6071 (2006).

6. D. Vermeulen, S. Selvaraja, P. Verheyen, G. Lepage, W. Bogaerts, P. Absil, D. van Thourhout, and G. Roelkens, Opt. Express 18, 18278 (2010).

7. X. Chen, C. Li, K. Y. Fung, M. Stanley, G. Lo, and H. K. Tsang, IEEE Photon. Technol. Lett. 22, 1156 (2010).

8. C. Li, H. Zhang, M. Yu, and G. Lo, Opt. Express 21, 7868 (2013).

9. L. Liu, M. Pu, K. Yvind, and J. M. Hvam, Appl. Phys. Lett. 96, 051126 (2010).

10. R. Halir, P. Cheben, J. Schmid, R. Ma, D. Bedard, S. Janz, D. $\mathrm{Xu}, \mathrm{A}$. Densmore, J. Lapointe, and Í. Molina-Fernández, Opt. Lett. 35, 3243 (2010).

11. P. Bienstman, "Rigorous and efficient modelling of wavelength scale photonic components," Ph.D. dissertation (University of Ghent, 2001).

12. D. Gao and Z. Zhou, Appl. Phys. Lett. 88, 163105 (2006).

13. R. Orobtchouk, A. Layadi, H. Gualous, D. Pascal, A. Koster, and S. Laval, Appl. Opt. 39, 5773 (2000).

14. Y. Ding, H. Ou, and C. Peucheret, Opt. Lett. 38, 1227 (2013).

15. H. Hu, H. Ji, M. Galili, M. Pu, H. C. H. Mulvad, K. Yvind, J. M. Hvam, P. Jeppesen, and L. K. Oxenløwe, "All-optical wavelength conversion of a high-speed RZ-OOK signal in a silicon nanowire," in Photonics Conference (IEEE, 2011), pp. 595-596.

16. W. S. Zaoui, M. F. Rosa, W. Vogel, M. Berroth, J. Butschke, and F. Letzkus, in European Conference and Exhibition on Optical Communication, OSA Technical Digest (Optical Society of America, 2012), paper Tu.1.E.2. 